\title{
Mode locking in discrete soliton dynamics under ac forces
}

\author{
P. J. Martínez \\ Departamento de Física Aplicada, Universidad de Zaragoza, 50009 Zaragoza, Spain \\ and Instituto de Ciencia de Materiales de Aragón, C.S.I.C.-Universidad de Zaragoza, 50009 Zaragoza, Spain \\ F. Falo, J. J. Mazo, and L. M. Floría \\ Departamento de Física de la Materia Condensada, Universidad de Zaragoza, 50009 Zaragoza, Spain \\ and Instituto de Ciencia de Materiales de Aragón, C.S.I.C.-Universidad de Zaragoza, 50009 Zaragoza, Spain
}

\author{
A. Sánchez \\ Grupo Interdisciplinar de Sistemas Complicados, Departamento de Matemáticas, Universidad Carlos III de Madrid, \\ 28911 Leganés, Spain \\ (Received 10 March 1997)
}

\begin{abstract}
We present here analytical arguments and numerical evidence for the existence of net directional motion of highly discrete sine-Gordon kinks under ac forces of zero average. We have also characterized the depinning of the oscillating kink under those circumstances, and analyzed the instability mechanisms of the phase-locked running solutions. Possible experimental relevance of this phenomenon in circular arrays of Josephson junctions is discussed. [S0163-1829(97)02726-4]
\end{abstract}

The discrete sine-Gordon equation (Frenkel-Kontorova model) appears as a mathematical description of various condensed-matter physical systems such as charge (or spin) density waves, arrays of superconducting Josephson junctions (JJA's), crystal dislocations, domain walls in magnetic and ferroelectric systems, etc. This ubiquity is not surprising for it describes, in an abstract way, a discrete elastic medium modulated by a periodic substrate potential, a paradigmatic notion in condensed-matter physics. As such, the study of the Frenkel-Kontorova (FK) model has provided much insight into the physical consequences of length and time scale competition in macroscopic systems. ${ }^{1-4}$

The equations of motion of the Frenkel-Kontorova chain submitted to additional damping and driving forces are, in dimensionless form,

$$
\ddot{u}_{j}+\alpha \dot{u}_{j}+\frac{K}{2 \pi} \sin \left(2 \pi u_{j}\right)=u_{j+1}-2 u_{j}+u_{j-1}+F,
$$

where $u_{j}$ is the phase of the $j$ th oscillator, $F$ is the driving force, $\alpha$ is the damping, and $K$ measures the strength of the substrate potential (so that it can be regarded as the discretization parameter). In the limit $K \rightarrow 0$ we recover the continuous sine-Gordon model. Aubry ${ }^{1}$ has shown the influence of discretization in the ground-state properties of the model. On the other hand, discreteness has dramatic effects in the dynamics of defects of the FK chain (kink or solitons). Let us mention two important observable effects which are immediate consequences of the discreteness. The first one is the emission of radiation of small-amplitude oscillations (linear waves or phonons) in the motion of defects. ${ }^{2,5,6}$ Recent studies of equations (1), with constant homogeneous force $F$, have provided a good quantitative explanation of experimental measurements of the current-voltage characteristics of circular arrays (rings) of underdamped Josephson junctions biased by constant currents. ${ }^{7,8}$ An important outcome from these comparisons between theory and experiments is the essential role played by the discrete character of the model and the finite number of degrees of freedom of the experimental system; in other words, essential aspects of the physical phenomena are lost when a continuum limit of equations (1) is taken. In particular, these experiments clearly confirm that a kink solution (a quantum of flux trapped in the JJA's), in the low-voltage region (i.e., low values of $F$ ), radiates small-amplitude oscillations which couple with its own motion giving new phase-locked steps in the measured characteristics. ${ }^{8}$

The second effect is the pinning of the kink to the underlying discrete lattice. ${ }^{1,4-6}$ The kink has to overcome an energy barrier [the so-called Peierls-Nabarro (PN) barrier] to start to move; otherwise the soliton stays trapped oscillating with a characteristic frequency, known as the PN frequency. This frequency appears in the gap of the linear waves spectrum and substitutes the zero frequency (Goldstone mode) associated with the translation invariance of the continuous system. In this situation, the kink is highly localized in the lattice and its dynamics look like the one of a particle moving in a periodic potential which strength is given by the PN energy.

In this paper we will study the underdamped dynamics of the kink when $F$ is a periodic function of time with zero average,

$$
F=F_{\mathrm{ac}} \cos \omega t
$$

The main result we report in this paper is that the kink can slide and move with net average velocity. This type of symmetry-breaking solutions occur for certain ranges of the parameter values $\left(K, \alpha, F_{\mathrm{ac}}\right.$, and $\left.\omega\right)$.

A useful way to obtain information on the overall behavior of the kink is to make use of the collective coordinate formalism. ${ }^{9}$ Following this procedure, the motion of the cen- 


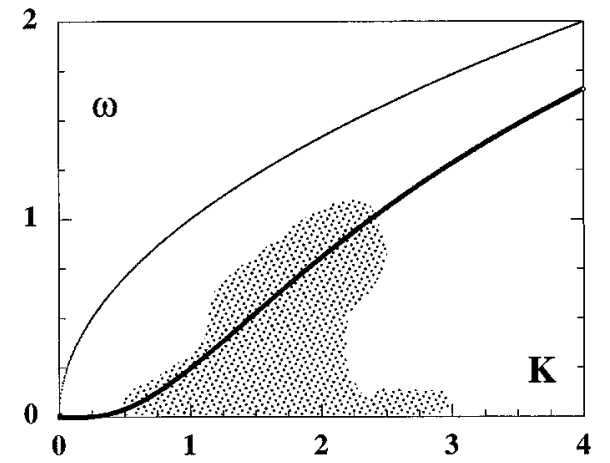

FIG. 1. Schematic phase diagram in the $\omega$ vs $K$ plane showing (dotted area) values of the parameters for which a mode-locking motion of the kink at some value of the intensity of the external force $F_{\mathrm{ac}}$ is found (here $\alpha=0.1$ ). Upper and lower curves show the phonon band gap and the Peierls-Nabarro frequency, respectively.

ter of mass of the kink is described by the equation of motion for an effective pendulum where the parameters are dressed by the influence of the nonlinear waves. This equation is known to possess running solutions under ac forces of zero average. We have checked that these types of solutions do exist for the kink in the Frenkel-Kontorova chain, by direct simulation of the complete equations of motion, confirming the prediction from the collective coordinate formalism. An interesting aspect of these solutions is that the velocity of the kink is determined by the external driving frequency, a parameter that the experimentalist can easily control.

In order to keep the analysis as close as possible to the experimental situations referred to above, ${ }^{8}$ let us consider a finite chain of $N$ particles with $u_{j+N}=u_{j}+N$. The spectrum of linear modes (phonons) is $\omega(q)=\sqrt{K+4 \sin ^{2} q / 2}$ where $q=2 \pi(m / N) \quad(m=0, \pm 1, \pm 2, \ldots)$. Discreteness (nonzero $K$ ) breaks the continuous translational invariance of the chain, pinning it to the substrate. The consequence is the appearance of a gap in the linear waves spectrum of the lattice $\omega_{g}=\min \omega(q)=\sqrt{K}$.

For a lattice of $N$ particles a (anti-) soliton is given by the following boundary condition: $u_{j+N}=u_{j}+N \pm 1$. With this boundary condition an eigenvalue corresponding to a localized mode appears in the gap. In the continuum limit $(K \rightarrow 0)$ this eigenvalue vanishes, a fact which corresponds to the unpinned character of the soliton in this limit. Figure 1 shows the eigenvalue associated with the localized mode for a soliton in a lattice of 80 particles. This eigenvalue remains (numerically) close to zero (sliding soliton) until $K \simeq 0.4$. For higher values of $K$ the soliton becomes more and more localized (i.e., its width decreases) and is pinned to the substrate (Peierls-Nabarro barrier ${ }^{1,4}$ ). The squared root of the eigenvalue defines the frequency of small oscillations of the soliton around its equilibrium position, the Peierls-Nabarro frequency $\Omega_{\mathrm{PN}}$.

Using the formalism developed in Refs. 9 and 10 we can find the effective equation of motion for the soliton center of mass (CM) by assuming a sine-Gordon profile for the discrete (anti-) soliton,

$$
u_{n}=n \pm \frac{2}{\pi} \tan ^{-1}\left\{\exp [n-X(t)] / l_{0}\right\}
$$

where $l_{0}$ stands for the soliton width. Such a parameter is directly related to $K$. We can derive a second-order differential equation for the soliton $\mathrm{CM}, X(t)$ :

$$
\ddot{X}(t)+\eta \dot{X}(t)-\frac{\Omega_{\mathrm{PN}}^{2}}{2 \pi} \sin (2 \pi X(t))=-\widetilde{F}_{\mathrm{ac}} \cos \omega t .
$$

Here we have averaged over the phonon degrees of freedom, which "dress" the parameters to give the following expressions:

$$
\eta=\alpha, \quad \frac{\Omega_{\mathrm{PN}}^{2}}{2 \pi}=\frac{\pi^{3}+2 \pi^{5} l_{0}^{2}}{6 l_{0}^{4} \sinh \left(\pi^{2} l_{0}\right)}, \quad \widetilde{F}_{\mathrm{ac}}=\frac{\pi^{2} l_{0}}{2} F_{\mathrm{ac}} .
$$

Equation (4) is the familiar expression for a damped and forced simple pendulum. Such an equation presents a rich variety of different solutions when the initial conditions and/or the parameters are varied. Of particular interest here are the so-called ${ }^{11,12}$ running solutions (appearing in pairs) which are attractors with nonzero average velocity $\langle\dot{X}(t)\rangle$. As Eq. (4) is obtained under the assumption (3) for the soliton shape and reducing drastically the number of degrees of freedom, the question arises whether or not these running solutions correspond to true solutions for the kink of the Frenkel-Kontorova chain (1). Mode-locking solutions of the pendulum would allow kink velocities controlled by the external frequency.

In order to check the prediction of the collective coordinate scheme we have simulated the dynamics of a soliton driven by a pure uniform ac driving force. A fourth-order Runge-Kutta method was used to integrate Eq. (1) with the appropriate boundary conditions. The lattice size was chosen to be much larger than the soliton width in order to avoid soliton-soliton interaction via boundary conditions. We have worked out the response of the chain as a function of $F_{\text {ac }}, \omega$, and $K$ for an intermediate value of damping $\alpha=0.1$ in which the collective coordinates scheme works well. In Fig. 1 we indicate the ranges of parameter values for which a coherent mode-locking motion of the soliton appears. We compare them with the frequency of phonons and the PN frequency. Figure 2 shows a typical response for fixed values of parameters $K$ and $\omega$ in the region of interest for high and low frequencies and a comparison with collective coordinate equation of motion. ${ }^{13}$ Next, we discuss these response curves.

Low-frequency response [Fig. 2(a)]: We analyze here the response for low values of $\omega$-approximately a half of the PN frequency. For low values of the strength $F_{\text {ac }}$ of the driving force, the soliton remains trapped in the bottom of a single PN well, oscillating with the driving frequency. As soon as the strength of the force reaches a critical value the soliton starts to jump to contiguous wells.

The Floquet analysis ${ }^{14}$ of periodic (pinned or moving) solutions gives insights into the mechanisms of this depinning of the kink under the action of just an ac force. This is to analyze the local stability matrix (small perturbation matrix) of such solutions generated along a period of the stable periodic attractor. An eigenvalue crossing the unit circle indicates the breaking of stability. In this case when the depinning instability occurs the largest eigenvalue (which is real) tends to +1 . This characteristic corresponds to a saddle-node 

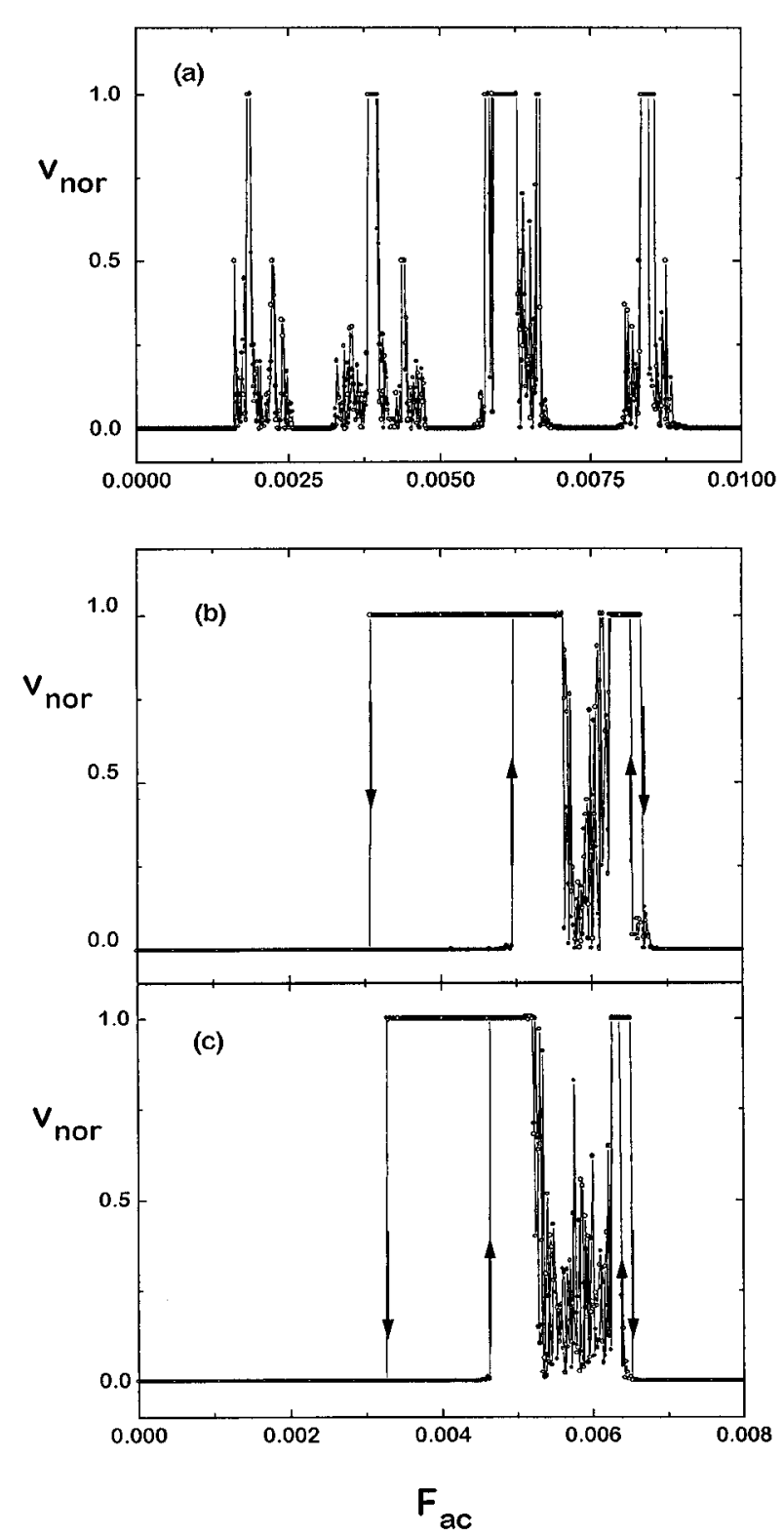

FIG. 2. $v_{\text {nor }}$ vs $F_{\text {ac }}\left(v_{\text {nor }}=2 \pi v_{\text {kink }} / \omega\right)$ for a $N=20$ lattice with a kink: (a) low-frequency regime $\omega=2 \pi \times 0.02$ and (b) highfrequency regime $\omega=2 \pi \times 0.04$. (c) shows the result of the collective coordinate formalism for the values of the parameters of (b). $\alpha=0.1, K=1.0, \Omega_{\mathrm{PN}}^{2}=0.06$, and $\widetilde{F}_{\mathrm{ac}} \simeq 5 F_{\mathrm{ac}}$.

bifurcation, which has associated type-I intermittencies. ${ }^{14}$ The eigenvector associated with this soft mode is an asymmetric "depinning mode" and it is localized in the soliton $\mathrm{CM}$. In the depinned phase (close enough to the critical depinning point) we observe intermittencies of type I: The soliton stays for a long time in the bottom of a well, suddenly jumps to another, and temporally remains in it [see Fig. $3(\mathrm{a})$. We can characterize the chaotic state as the result of the competition of (at least) three "metastable attractors": the kink trapped in a well and running in two opposite directions. Hops between these states determine the dynamical state in this region. The frequency of these jumps increases with $F_{\text {ac }}$ until a diffusive motion is reached [Fig. 3(b)]. Further increment of the control parameter causes the residence time in the running attractors to increase. Finally a mode-
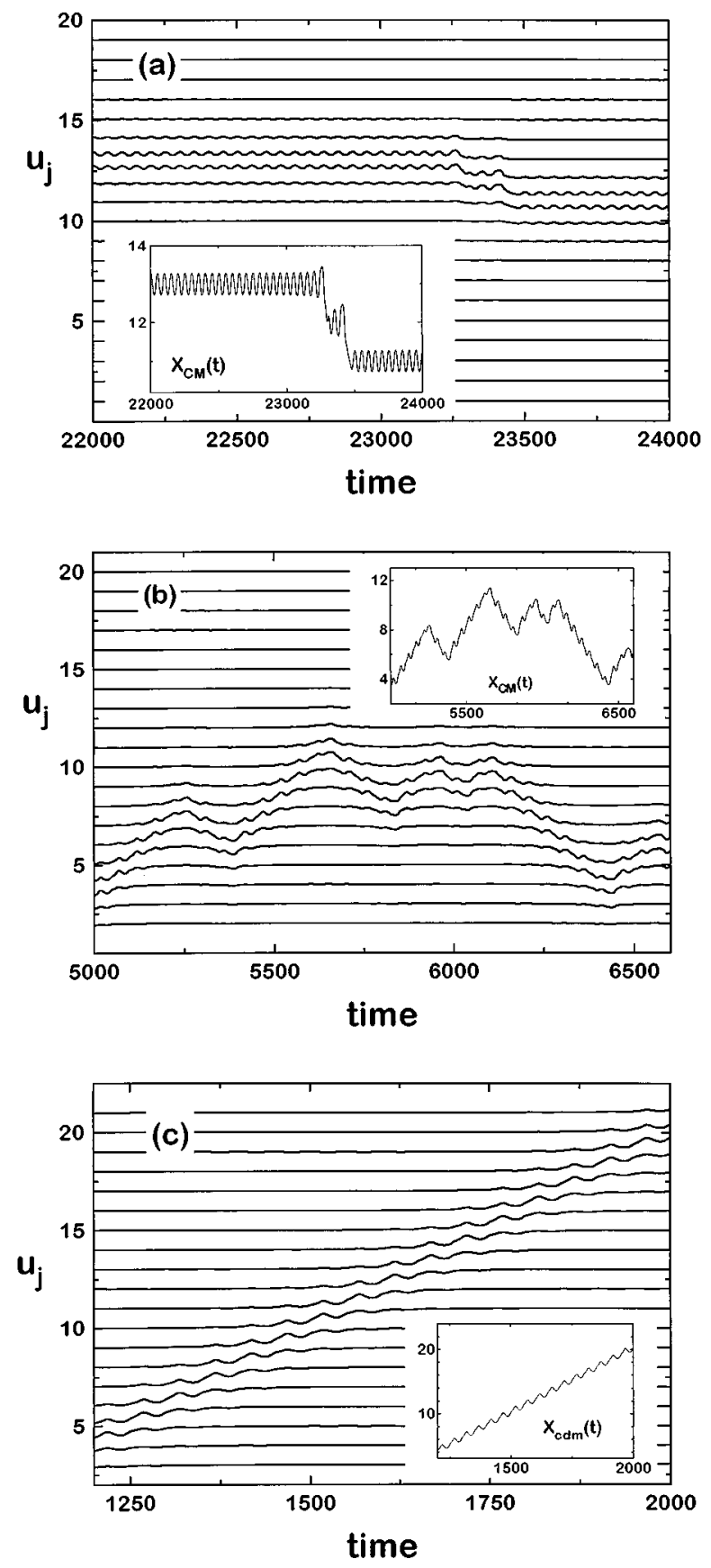

FIG. 3. Three different types of possible motion of the soliton in the lattice corresponding to three different points of the curve shown in Fig. 2(a). (a) Intermittent motion of the soliton $\left(F_{\text {ac }}=0.00163115\right)$. (b) Diffusive motion of the kink $\left(F_{\mathrm{ac}}=0.0018\right)$. (c) Mode-locking motion of the kink corresponding to a step of resonant velocity, $v_{\text {nor }}=1 \quad\left(F_{\text {ac }}=0.00185\right)$. $\omega=2 \pi \times 0.02, \alpha=0.1$, and $K=1.0$. The insets show the motion of the $\mathrm{CM}$ of the kink.

locking regime is achieved in which the averaged velocity is exactly given by

$$
v_{\mathrm{kink}}=\frac{p}{q} \frac{\omega}{2 \pi}
$$

where $\omega$ is the frequency of driving force and $p$ and $q$ are some coprime integer numbers. The kink steadily moves in a 
direction depending on the initial conditions [Fig. 3(c)]. An important point to emphasize is that the amplitude of the ac force is quite below (by approximately an order of magnitude) the depinning dc force for the same parameters of the model. At these values of the external frequency we do not observe hysteretic behavior in the curves so there is not coexistence between pinned and unpinned attractors. However, the kink motion within the mode-locking step is not periodic but substantially more complex. It undergoes a cascade of period-doubling bifurcations with only small windows of periodicity.

High-frequency response [Fig. 2(b)]: For $\omega$ close to the $\mathrm{PN}$ frequency the depinning behavior is quite different. Prior to depinning, the symmetric oscillating state of the soliton within a well breaks in two states in which it oscillates around one of the two sides of the well, depending on initial conditions. This instability is also driven by an eigenvalue which crosses the unit circle through +1 and the corresponding eigenvector is localized. By increasing $F_{\text {ac }}$ these new states become unstable and a new cascade of double-period bifurcations appears. Once this confined motion is chaotic, the kink can move and a mode-locking step is observed. A significant fact, in this region, is the coexistence of running and pinned stable solutions for the same $F_{\text {ac }}$ value. The consequence is a hysteresis loop in the response. The behavior in the upper branch is similar to that observed in the modelocking step at low frequency. The instability of this running state (by lowering $F_{\text {ac }}$ ) also corresponds to a localized mode associated with a $(+1)$ eigenvalue.

These curves have been obtained for fixed $K=1$. For higher $K$ values $(\simeq 4)$ we cannot observe coherent kink motion since the chaotic dynamics of the whole lattice, dominates.

Finally we discuss the role of damping in the dc motion of the soliton. For overdamped dynamics, the "nonpassing rule" of Middleton ${ }^{15}$ applies: for ac fields and convex interparticle interactions Middleton has proved that the steadystate velocity of the chain is unique and independent of the initial conditions. This forbids the symmetry-broken solutions in the motion found here. On the other hand, for very low damping the collective coordinate map cannot account for the behavior of the many-particle system. To have a complete description of the system, we need to include the equations of motion for the phonons. We have found some parameter values for which Eq. (4) has mode-locking solutions in which the simulations of the complete system show the instability of the kink motion by the emission of a large amount of phonons. However, we have found that for intermediate values $(\alpha=0.05-0.5)$ the existence of mode locking holds and we can find parameter values for the steps.

In summary, we have shown that a kink in a discrete Frenkel-Kontorova chain can steadily move sustained by only an ac force. This phenomenon can be understood in the context of mapping the kink motion into a single particle moving in a periodic potential. Given the generality of the methods used here, we expect that this behavior can be observed in the dynamics of localized defects in other nonlinear lattices (e.g., the continuum and discrete $\phi^{4}$ models).

This model is easily experimentally feasible, for instance in a ring of Josephson-junctions ${ }^{8}$ with trapped magnetic flux. For the parameter of one of the samples given there, we find $K=0.5, \Omega_{\mathrm{PN}}=0.04$, and $\alpha=0.08$. This system is moderately discrete (see Fig. 1) and one can expect finite-size effects in the flux dynamics. Simulations with these parameters and rings of $N=8$ and $N=20$ lattice sites, show mode-locking steps for a very few values of the external frequency. Another experimental system, in which the $K$ parameter is reasonably well controlled is a two-parallel array of JJ's (a ladder). ${ }^{16}$ Although the dynamical behavior of vortex does not follow the discrete sine-Gordon equation, preliminary computer simulations confirm the validity of the above results.

We acknowledge A. R. Bishop and J. L. Marín for many useful suggestions on this work. Financial support from DGES (PB95-0797) and CICYT (MAT95-0325), Spain is acknowledged.
${ }^{1} \mathrm{~S}$. Aubry, in Structures et Instabilités, edited by C. Godrèche (Editions de Physique, Les Ulis, France, 1985).

${ }^{2}$ S. Aubry and L.de Seze, Festkoerperprobleme XXV, 59 (1985).

${ }^{3}$ S. N. Coppersmith and D. S. Fisher, Phys. Rev. B 28, 2566 (1983).

${ }^{4}$ L. M. Floría and J. J. Mazo, Adv. Phys. 45, 505 (1996).

${ }^{5}$ M. Peyrard and M. D. Kruskal, Physica D 14, 88 (1984).

${ }^{6}$ R. Boesch, C. R. Willis, and M. El-Batanouny, Phys. Rev. B 40, 2284 (1989).

${ }^{7}$ A. V. Ustinov, M. Cirillo, and B. A. Malomed, Phys. Rev. B 47, 8357 (1993).

${ }^{8}$ S. Watanabe, H. S. J. van der Zant, S. H. Strogatz, and T. P. Orlando, Physica D 97, 429 (1996).

${ }^{9}$ C. R. Willis, M. El-Batanouny, and P. Stancioff, Phys. Rev. B 33, 1904 (1986).

${ }^{10}$ M. K. Sayadi and J. Pouget, Physica D 55, 259 (1992).
${ }^{11}$ E. G. Gwinn and R. M. Westervelt, Phys. Rev. A 33, 4143 (1986).

${ }^{12}$ D. D'Humieres, M. R. Beasley, B. A. Huberman, and A. Libchaber, Phys. Rev. A 26, 3483 (1982).

${ }^{13}$ In order to compare the simulations of the complete chain [Eq. (1)] with that of Eq. (4) we obtain $l_{0}$ by equating the numerically computed PN frequency, $\Omega_{\mathrm{PN}}$, with the expression in the collective coordinate results, Eq. (5). This allows us to calculate the other parameters of the pendulum equation.

${ }^{14} \mathrm{P}$. Manneville, Dissipative Structures and Weak Turbulence (Academic, New York, 1990).

${ }^{15}$ A. A. Middelton, Phys. Rev. Lett. 68, 670 (1992).

${ }^{16}$ L. M. Floría, J. L. Marín, P. J. Martínez, F. Falo, and S. Aubry, Europhys. Lett. 36, 539 (1996); J. J. Mazo and J. C. Ciria, Phys. Rev. B 54, 16068 (1996). 